\section{Efectos del ejercicio físico de alta intensidad y sobrecarga en parámetros de salud metabólica en mujeres sedentarias, pre-diabéticas con sobrepeso u obesidad}

\author{
CRISTIAN ÁLVAREZ $Z^{1, \mathrm{a}}$, RODRIGO RAMÍREZ ${ }^{2, \mathrm{~b}}$, MARCELO FLORES $^{3,4, \mathrm{c}}$, \\ CECIL ZÚÑIGA ${ }^{5, \mathrm{~d}}$, CARLOS A. CELIS-MORALES ${ }^{6, \mathrm{e}}$
}

\section{Effect of sprint interval training and resistance exercise on metabolic markers in overweight women}

Background: Physical activity is associated with an improvement in cardiovascular health, however there is a paucity of information about the effects of sprint interval training on individuals with high metabolic risk. Aim: To determine the effects of three exercise programs on anthropometric and metabolic markers in overweight, sedentary and prediabetic women. Material and Methods: Forty three women were ascribed to four groups matched by body mass index and body fat: high intensity intervals (HIT, $n=12)$, resistance $(R, n=8)$, combined group $(H I T+R, n=10)$ and control group (CG, $n=13$ ). Participants completed 12 weeks of exercise intervention. Body mass index, waist circumference, percentage of fat mass measured by impedanciometry, blood pressure, fasting glucose, insulin and homeostasis model assessment for insulin resistance (HOMA $\left.{ }_{I R}\right)$ and fitness assessed using the two $\mathrm{km}$ walk test were measured at baseline and after the training period. Results: No changes in anthropometric and body composition variables were observed. However, in HIT and $R$ groups, significant reductions were observed on fasting glucose (5.4 and 16.6\% respectively), insulin (18.6 and $43.4 \%$ respectively) and $\mathrm{HOMA}_{I R}$ (24.1 and 55.4\% respectively), 72 hours after the intervention. No significant changes were found for the observed values in the combined and control groups. Conclusions: HIT and resistance training improve glycemic control and insulin sensitivity in females with a high metabolic risk.

(Rev Med Chile 2012; 140: 1289-1296).

Key words: Exercise; Insulin Resistance; Obesity; Sedentary Lifestyle.

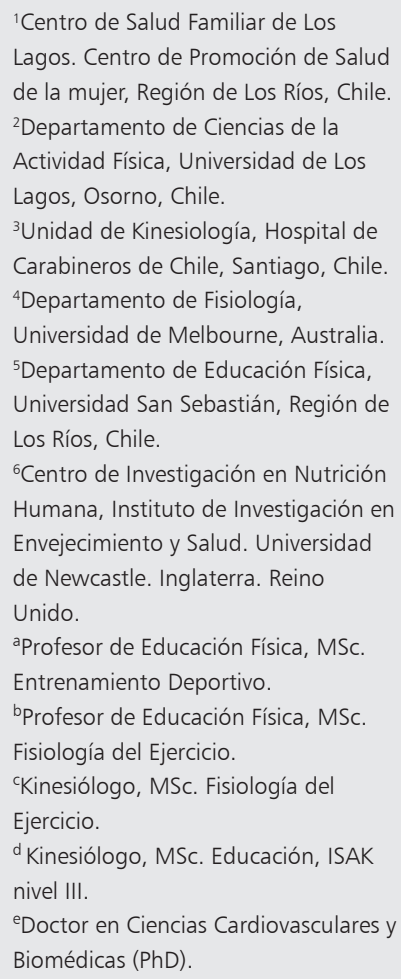

Financiamiento: Proyecto financiado por el concurso de Fondos de Investigación Internos, Universidad de Los Lagos, Osorno, Chile, otorgado a Ramírez R y Alvarez C. No se declaran conflicto de intereses entre las fuentes de financiamiento y el presente estudio.

Recibido el 6 de marzo de 2012, aceptado el 8 de junio de 2012 .

Correspondencia a

Dr. Carlos Celis Morales, Biomedical Research Building Campus for Ageing and Vitality Newcastle Upon Tyne, NE4 5PL. UK Fono: +44(0)191248 1141

Email: carlos.celis@newcastle.ac.uk
4 1 sobrepeso y la obesidad son estados que se asocian al desarrollo de insulino resistencia (IR), diabetes tipo 2 (DT2) y enfermedad cardiovascular $(\mathrm{ECV})^{1-3}$. Adicionalmente, el sedentarismo ha sido señalado como otro factor relevante en el aumento de estas patologías ${ }^{3-5}$. En
Chile, 93\% de las mujeres son sedentarias y $64 \%$ presentan sobrepeso u obesidad y actualmente las ECV constituyen la principal causa de muerte en el país ${ }^{6,7}$.

A pesar de la asociación entre la práctica de actividad física $(\mathrm{AF})$ y la reducción de $\mathrm{ECV}^{8}$, los 
niveles de AF permanecen bajos en la población? Esto ha llevado a proponer nuevas recomendaciones de AF para población adulta, que reducen los tradicionales 150 min de AF por semana a 20 min de AF de mayor intensidad y con menor duración (ej. 3 veces por semana $)^{10,11}$. Este cambio podría ser un importante paso para incrementar los niveles de AF, ya que la falta de tiempo ha sido identificada como una de las principales barreras para la práctica de AF en la población adulta ${ }^{12}$. Sin embargo, en relación a los efectos de esta nueva práctica de $\mathrm{AF}$ en personas con riesgo metabólico es limitada. Teniendo en cuenta los niveles de sedentarismo, obesidad, DT2 y ECV en Chile, se hace necesario diseñar, y evaluar otros modelos de AF, que sean factibles de implementarse en servicios y programas de salud locales a lo largo del país. Por ende, el objetivo de este trabajo fue evaluar el impacto de 3 programas de AF sobre parámetros antropométricos y de salud cardiovascular en mujeres sedentarias, pre-diabéticas y con sobrepeso.

\section{Material y Métodos}

\section{Participantes}

Cuarenta y tres mujeres participaron en el estudio $(n=43)$, las cuales fueron reclutadas en el Centro de Salud Familiar de Los Lagos (CESFAM), Región de Los Ríos, Chile. El diseño del estudio corresponde a un estudio experimental con muestreo aleatorio simple. Los participantes fueron distribuidos en cuatro grupos: programa de intervalos (PI), programa de sobrecarga (PS), programa mixto (PI+PS) y grupo control (GC). La asignación de los participantes en cada grupo fue pareada según IMC y \% masa grasa. El tamaño de la muestra se estimó utilizando cambios observados en insulina plasmática (delta $=2,16$ $\mathrm{mU} / \mathrm{l} ; \mathrm{d} . \mathrm{s}=1,38)$ en un grupo intervenido con diferentes programas de ejercicio físico ${ }^{13}$. Un total de 9 participantes por grupo otorga una potencia de $80 \%$ y un $\alpha$ de 0,05 .

Los criterios de inclusión fueron: a) ser sedentaria (ejercicio $\leq 30 \mathrm{~min} /$ semana), b) $\mathrm{IMC}>25,0$ $\mathrm{kg} / \mathrm{m}^{2}$, c) nivel de glicemia $100 \mathrm{a} \leq 125 \mathrm{mg} / \mathrm{dl}^{14}$. Los criterios de exclusión fueron: a) antecedentes de enfermedad ósteo-articular, isquémica, arritmias, taquicardias y/o enfermedad pulmonar obstructiva crónica; b) con tratamiento farmacológico para alteraciones metabólicas. Este estudio se realizó considerando la Declaración de Helsinki y fue aprobado por los comités de ética del CESFAM Los Lagos, Osorno, Chile. Todos los participantes dieron su consentimiento informado por escrito previo a la intervención.

\section{Procedimientos}

Se determinó peso corporal, masa grasa (MG) y masa muscular (MM), utilizando una balanza digital de bioimpedancia de pies y manos (OMRON ${ }^{\circledR}$, Modelo HBF-INT). La talla se midió con un tallímetro de $0,1 \mathrm{~cm}$ de precisión (Health o Meter ${ }^{\circledR}$, USA). El IMC se calculó dividiendo el peso corporal por la talla elevada al cuadrado $\left(\mathrm{kg} / \mathrm{m}^{2}\right)$ y sobrepeso fue definido como IMC $>25 \mathrm{~kg} / \mathrm{m}^{2}$. El perímetro de cintura se midió justo por encima de la línea de la cresta ilíaca ${ }^{15}$ con una cinta inextensible y precisión de $0,1 \mathrm{~cm}$ (Hoechstmass ${ }^{\circledR}$, West Germany 1-150 cm).

La presión arterial se determinó con un monitor digital (OMRON, modelo HEM-742INT). Cada participante permaneció en posición sentado al menos 15 min previos a la medición, reportándose únicamente la media de tres mediciones. Se obtuvieron muestras de sangre en ayunas $(4 \mathrm{ml})$ entre las 8:00 AM y 11:00 AM (Pre intervención, 24 h y 72 h Post intervención). Glicemia se determinó con método enzimático (Trinder, Genzyme Diagnostics, Canada) e insulina fue determinada mediante RIA (DPC, Los Angeles, CA). Insulino resistencia se determinó con $\mathrm{HOMA}_{\mathrm{IR}}{ }^{16}$.

Se aplicó el test UKK para determinar la capacidad de traslación bípeda ${ }^{17}$. Cuestionarios sociodemográfico y de salud fueron aplicados para determinar edad, antecedentes de tratamiento farmacológico, historia familiar de enfermedades señaladas en los criterios de inclusión y exclusión.

\section{Diseño de los programas de intervención}

Programas de Ejercicio físico intervalado (PI): consistió en realizar carreras y pausas de recuperación de manera intervalada hasta completar un volumen de 20 min de AF por día y 2 veces por semana. En cada sesión los participantes completaron 7 intervalos de carreras de alta intensidad (> 85\% de la frecuencia cardiaca máxima (220 Edad). Cada intervalo tuvo una duración de $20 \mathrm{~s}$ y se incrementó en 2 s (10\%) cada 2 semanas, mientras que el intervalo de recuperación de 120 s, disminuyó en $5 \mathrm{~s}(\sim 4 \%)$ cada 2 semanas (Tabla 1). 
Ejercicio físico en mujeres con riesgo metabólico - C. Álvarez et al

Tabla 1. Descripción de los protocolos de ejercicio físico utilizados en la intervención

\begin{tabular}{|llll|}
\hline & $\begin{array}{l}\text { PI } \\
(\mathbf{n}=\mathbf{1 2})\end{array}$ & $\begin{array}{l}\text { PS } \\
(\mathbf{n}=\mathbf{8})\end{array}$ & $\begin{array}{l}\text { PI + PS } \\
(\mathbf{n}=\mathbf{1 0})\end{array}$ \\
\hline Intensidad & $100 \%$ FCM & "Hasta la fatiga" & $100 \%$ FCM + Fatiga \\
\hline Duración Intervalo (s) & 20 a 30 & - & 20 a 30 \\
\hline Número de Intervalos & 7 & - & 7 \\
\hline Recuperación (s) & 120 a 150 & 120 & 120 a 150 \\
\hline Método de Recuperación & Caminando & Estática & Estática \\
Volumen (min/día) & 20 & 45 & $20-45$ \\
\hline Volumen (min/sem) & 60 & 90 & 150 \\
\hline Frecuencia (días/sem) & 3 & 2 & 5 \\
\hline Equipamiento necesario & - & Barras y pesos libres & Barras y pesos libres \\
\hline
\end{tabular}

Grupos de intervención son descritos como: Programa de Intervalos (PI), Programa de Sobrecarga (PS), Grupo combinado (PI+PS), Grupo Control (GC). Frecuencia cardiaca Máxima (FCM).

Programa de Ejercicio físico de sobrecarga (PS): consistió en realizar 5 ejercicios diferentes de sobrecarga (sentadilla, flexo-extensión de bíceps, flexo-extensiones de tobillo, flexo-extensión de hombros y flexo-extensiones de codo). Cada ejercicio se realizó durante 1 minuto (alcanzando el fallo muscular) y se repitió 3 veces por sesión, con pausas de 2 min de recuperación entre cada serie $^{18}$ (Tabla 1).

Programa de Ejercicio físico combinado (PI+PS): consistió en realizar ambos programas de ejercicios en días no consecutivos, realizando un total de 5 días de ejercicio físico a la semana (Tabla 1).

\section{Dieta, Actividad Física y Adherencia}

Se instruyó a todos los participantes no cambiar su dieta y patrones de AF durante el transcurso de la intervención. La adherencia presentada por los tres grupos fue PI (85\%), PS (95\%) y $\mathrm{PI}+\mathrm{PS}(74 \%)$.

\section{Estadística}

Los datos son presentados como media \pm desviación estándar. Se aplicó el test de Shapiro-Wilk para determinar si las variables correspondían a una distribución normal. Diferencias entre Pre y Post intervención se determinaron con test de $\mathrm{t}$ de Student para muestras relacionadas. Para determinar si existían diferencias significativas en variables cuantitativas entre los cuatro grupos Pre intervención se aplicó análisis ANOVA de dos vías y para determinar diferencias entre pre-test, post-test $24 \mathrm{~h}$ y post-test $72 \mathrm{~h}$ se aplicó ANOVA de una vía, el test Bonferoni fue utilizado para detectar donde se encontraban estas diferencias. Los análisis fueron ajustados a edad y adherencia al programa de intervención. Todos los análisis fueron realizados en SPSS (versión 19). El nivel de significación aceptado fue $\mathrm{p}<0,05$.

\section{Resultados}

En la Tabla 2 se describen las variables antropométricas estratificadas por programa de AF Pre y Post intervención. La edad y características antropométricas (peso, talla, IMC, perímetro de cintura y $\%$ de masa grasa) de los participantes no presentaron diferencias significativas entre grupos previo a la intervención.

En relación a las variables metabólicas (Tabla 3), la presión arterial sistólica presentó diferencias entre los grupos previo a la intervención $(\mathrm{p}<0,001)$, siendo significativamente mayor en el grupo PI en comparación al grupo PI+PS y al grupo control. No obstante, la presión arterial diastólica, test cardio-respiratorio UKK, glicemia, insulinemia y $\mathrm{HOMA}_{\mathrm{IR}}$ no presentaron diferencias significativas entre grupos previo a la intervención (Tabla 2).

Posterior a 12 semanas de intervención las variables antropométricas no mostraron cambios significativos entre los grupos intervenidos. En el test UKK de condición cardio-respiratoria, los grupos PI y PI+PS redujeron significativamente 
Tabla 2. Características antropométricas y de condición física de los participantes Pre y Post intervención estratificado por grupo

\begin{tabular}{|c|c|c|c|c|c|c|}
\hline Variable & Test & $\begin{array}{c}P^{a} \\
(n=12)\end{array}$ & $\begin{array}{c}\text { PS }^{b} \\
(n=8)\end{array}$ & $\begin{array}{l}P I+P S^{c} \\
(n=10)\end{array}$ & $\begin{array}{c}\text { GC }^{d} \\
(n=13)\end{array}$ & $\mathbf{P}_{\text {interacción }}$ \\
\hline Edad (años) & Pre & $39,2 \pm 9,5$ & $33,9 \pm 9,3$ & $43,3 \pm 8,1$ & $40,1 \pm 11,4$ & 0,255 \\
\hline Talla (m) & $\begin{array}{l}\text { Pre } \\
\text { Post } \\
\mathrm{p}_{\text {(Pre-Post) }}\end{array}$ & $\begin{array}{c}1,55 \pm 0,05 \\
1,55 \pm 0,04 \\
p=0,978\end{array}$ & $\begin{array}{c}1,58 \pm 0,05 \\
1,58 \pm 0,05 \\
p=0,999\end{array}$ & $\begin{array}{c}1,55 \pm 0,04 \\
1,54 \pm 0,04 \\
p=0,997\end{array}$ & $\begin{array}{c}1,57 \pm 0,05 \\
1,57 \pm 0,03 \\
p=0,998\end{array}$ & 0,417 \\
\hline Peso (kg) & $\begin{array}{l}\text { Pre } \\
\text { Post } \\
\mathrm{p}_{\text {(Pre-Post) }}\end{array}$ & $\begin{array}{l}70,3 \pm 6,6 \\
69,7 \pm 6,7 \\
p=0,832\end{array}$ & $\begin{array}{c}74,6 \pm 17,9 \\
74,6 \pm 16,8 \\
p=0,999\end{array}$ & $\begin{array}{l}69,8 \pm 7,4 \\
69,2 \pm 7,5 \\
p=0,853\end{array}$ & $\begin{array}{c}69,8 \pm 10,5 \\
70,2 \pm 10,5 \\
p=0,920\end{array}$ & 0,917 \\
\hline IMC $\left(\mathrm{kg} / \mathrm{m}^{2}\right)$ & $\begin{array}{l}\text { Pre } \\
\text { Post } \\
\text { P }_{\text {(Pre-Post) }}\end{array}$ & $\begin{array}{l}29,3 \pm 3,3 \\
29,1 \pm 3,2 \\
p=0,893\end{array}$ & $\begin{array}{l}29,4 \pm 5,5 \\
29,4 \pm 5,1 \\
p=0,996\end{array}$ & $\begin{array}{c}29,1 \pm 2,9 \\
28,8 \pm 2,9 \\
p=0,834\end{array}$ & $\begin{array}{c}28,3 \pm 3,5 \\
28,5 \pm 3,4 \\
p=0,891\end{array}$ & 0,915 \\
\hline P.Cintura $(\mathrm{cm})$ & $\begin{array}{l}\text { Pre } \\
\text { Post } \\
\text { P (Pre-Post) }\end{array}$ & $\begin{array}{c}101,1 \pm 6,4 \\
98,5 \pm 6,7 \\
p=0,319\end{array}$ & $\begin{array}{c}100,6 \pm 15,5 \\
98,5 \pm 14,5 \\
p=0,789\end{array}$ & $\begin{array}{c}98,2 \pm 5,7 \\
94,5 \pm 6,3 \\
p=0,089\end{array}$ & $\begin{array}{c}95,7 \pm 9,2 \\
96,6 \pm 9,2 \\
p=0,802\end{array}$ & 0,540 \\
\hline Masa grasa (\%) & $\begin{array}{l}\text { Pre } \\
\text { Post } \\
\text { p (Pre-Post) }\end{array}$ & $\begin{array}{c}44,0 \pm 3,9 \\
44,2 \pm 3,6 \\
p=0,877\end{array}$ & $\begin{array}{c}43,1 \pm 5,6 \\
42,5 \pm 5,8 \\
p=0,851\end{array}$ & $\begin{array}{c}43,5 \pm 4,2 \\
43,0 \pm 5,2 \\
p=0,800\end{array}$ & $\begin{array}{c}42,9 \pm 4,9 \\
43,7 \pm 6,5 \\
p=0,722\end{array}$ & 0,898 \\
\hline Test UKK (min) & $\begin{array}{l}\text { Pre } \\
\text { Post } \\
\text { P(Pre-Post) }\end{array}$ & $\begin{array}{c}21,9 \pm 1,1 \\
20,2 \pm 1,03 \\
p=0,0008\end{array}$ & $\begin{array}{l}21,3 \pm 0,8 \\
20,4 \pm 1,0 \\
p=0,066\end{array}$ & $\begin{array}{l}20,2 \pm 0,9 \\
18,5 \pm 0,9 \\
p=0,0009\end{array}$ & $\begin{array}{l}21,6 \pm 0,9 \\
21,1 \pm 0,8 \\
p=0,391\end{array}$ & 0,129 \\
\hline
\end{tabular}

Datos presentados como media \pm Desviación estándar. Grupos de intervención son descritos como: Programa de Intervalos (PI), Programa de Sobrecarga (PS), Grupo combinado (PI+PS), Grupo Control (GC). P er fue calculado con 2-way ANOVA para determinar si existían diferencias significativas entre grupos Pre-intervención. Diferencias significativas entre Pre y Post intervención fueron determinadas con t-test para muestras pareadas. Los análisis fueron ajustados por edad y asistencia al programa Nivel de significancia fue aceptado a $p<0,05$.

(a)

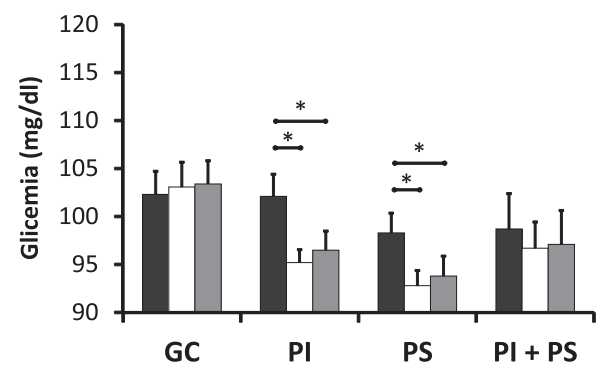

(c)

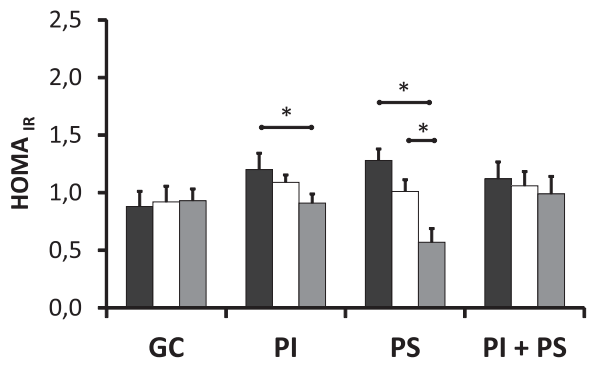

(b)

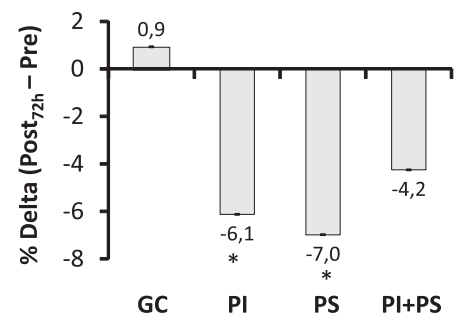

(d)

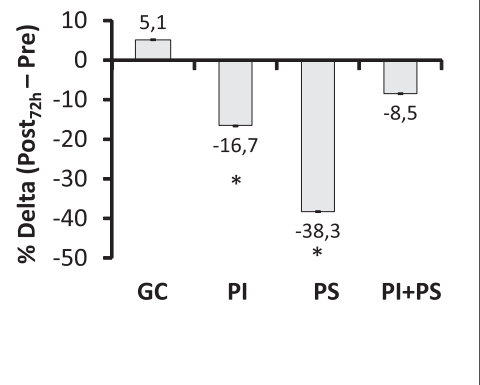

Figura 1. Cambios en Glicemia y $\mathrm{HOMA}_{\mathbb{R}}$ posterior a 12 semanas de intervención. Datos presentados como media \pm error típico de la media. Gráficos a y c muestran los niveles de glicemia e insulino resistencia pre intervención, 24 h y 72 h post intervención. Gráficos b y d muestran el $\%$ de reducción promedio (Post $_{72 h}$-Pre) en glicemia y $\mathrm{HOMA}_{\mathbb{R}}$ post intervención. Grupos son señalados como (PI: Programa de Intervalos, PS: Programa de Sobrecarga, PI + PS: Grupo combinado y GC: Grupo Control). Diferencias significativas entre pre, 24 h post y $72 \mathrm{~h}$ post fueron determinadas con ANOVA de una vía. Los modelos fueron ajustados por edad y asistencia al programa. Valores de significancia son señalados como ${ }^{*} p<0,05,{ }^{*} p<0,01$. 
Ejercicio físico en mujeres con riesgo metabólico - C. Álvarez et al

Tabla 3. Características metabólicas de los participantes Pre y Post intervención estratificado por grupo

\begin{tabular}{|c|c|c|c|c|c|c|}
\hline Variable & Test & $\begin{array}{c}P^{\mathrm{a}} \\
(n=12)\end{array}$ & $\begin{array}{c}\text { PS }^{b} \\
(n=8)\end{array}$ & $\begin{array}{l}P I+P S^{c} \\
(n=10)\end{array}$ & $\begin{array}{c}\text { GC }^{d} \\
(n=13)\end{array}$ & $\mathbf{P}_{\text {interacción }}$ \\
\hline PAS $(\mathrm{mm} / \mathrm{Hg})$ & $\begin{array}{l}\text { Pre } \\
\text { Post } \\
\text { p (Pre-Post) }\end{array}$ & $\begin{array}{c}138,5 \pm 17,1 \mathrm{cdd} \\
125,5 \pm 11,9 \\
p=0,043\end{array}$ & $\begin{array}{c}124,1 \pm 8,6 \\
116,5 \pm 16,1 \\
p=0,258\end{array}$ & $\begin{array}{c}117,4 \pm 12,9^{a} \\
113,4 \pm 10,2 \\
p=0,453\end{array}$ & $\begin{array}{c}120,1 \pm 10,2^{a} \\
121,1 \pm 7,6 \\
p=0,763\end{array}$ & 0,001 \\
\hline $\mathrm{PAD}(\mathrm{mm} / \mathrm{Hg})$ & $\begin{array}{l}\text { Pre } \\
\text { Post } \\
\mathrm{p}_{\text {(Pre-Post) }}\end{array}$ & $\begin{array}{l}81,9 \pm 9,7 \\
76,6 \pm 9,0 \\
p=0,183\end{array}$ & $\begin{array}{c}80 \pm 17,2 \\
74,1 \pm 6,3 \\
p=0,379\end{array}$ & $\begin{array}{c}70,2 \pm 12,9 \\
68,1 \pm 10,5 \\
p=0,696\end{array}$ & $\begin{array}{c}74,4 \pm 10,1 \\
75,4 \pm 9,1 \\
p=0,776\end{array}$ & 0,148 \\
\hline Glicemia (mg/dl) & $\begin{array}{l}\text { Pre } \\
\text { Post }{ }_{24 h r} \\
\text { p(Pre-Post 24hr) } \\
\text { Post } 72 h r \\
\text { p(Pre-Post 24hr) }\end{array}$ & $\begin{array}{c}102,1 \pm 9,2 \\
95,2 \pm 12,8 \\
p=0,011 \\
96,5 \pm 12,7 \\
p=0,024\end{array}$ & $\begin{array}{c}100,3 \pm 12,8 \\
92,8 \pm 9,6 \\
p=0,019 \\
93,8 \pm 5,6 \\
p=0,038\end{array}$ & $\begin{array}{c}101,2 \pm 5,2 \\
96,7 \pm 4,3 \\
p=0,369 \\
97,1 \pm 6,1 \\
p=0,088\end{array}$ & $\begin{array}{c}102,3 \pm 8,7 \\
103,1 \pm 9,3 \\
p=0,830 \\
103,4 \pm 8,8 \\
p=0,756\end{array}$ & 0,646 \\
\hline Insulina (mU/ml) & $\begin{array}{l}\text { Pre } \\
\text { Post }{ }_{24 h r} \\
\text { p (Pre-Post 24hr) }_{\text {Post }} \text { 72hr } \\
\text { p(Pre-Post 24hr) }\end{array}$ & $\begin{array}{l}4,67 \pm 3,1 \\
4,03 \pm 5,1 \\
p=0,189 \\
3,80 \pm 2,8 \\
p=0,041\end{array}$ & $\begin{array}{l}4,86 \pm 4,6 \\
4,28 \pm 4,1 \\
p=0,196 \\
2,75 \pm 0,9 \\
p=0,002\end{array}$ & $\begin{array}{l}4,53 \pm 4,8 \\
4,30 \pm 5,0 \\
p=0,530 \\
4,01 \pm 4,1 \\
p=0,192\end{array}$ & $\begin{array}{l}3,60 \pm 1,6 \\
3,70 \pm 1,7 \\
p=0,879 \\
3,75 \pm 1,6 \\
p=0,824\end{array}$ & 0,855 \\
\hline $\mathrm{HOMA}_{\mathbb{I R}}$ & $\begin{array}{l}\text { Pre } \\
\text { Post }{ }_{24 h r} \\
\text { p (Pre-Post 24hr) }_{\text {Post }} \text { 72hr } \\
\text { p(Pre-Post 24hr) }\end{array}$ & $\begin{array}{l}1,20 \pm 0,8 \\
1,09 \pm 1,2 \\
p=0,190 \\
0,91 \pm 0,7 \\
p=0,037\end{array}$ & $\begin{array}{l}1,28 \pm 1,4 \\
1,01 \pm 1,1 \\
p=0,184 \\
0,57 \pm 0,2 \\
p=0,009\end{array}$ & $\begin{array}{l}1,12 \pm 1,2 \\
1,06 \pm 1,0 \\
p=0,321 \\
0,99 \pm 1,1 \\
p=0,056\end{array}$ & $\begin{array}{l}0,88 \pm 0,4 \\
0,92 \pm 0,4 \\
p=0,823 \\
0,93 \pm 0,4 \\
p=0,790\end{array}$ & 0,824 \\
\hline
\end{tabular}

Datos presentados como promedio \pm Desviación estándar. Grupos de intervención son descritos como: Programa de Intervalos (PI), Programa de Sobrecarga (PS), Grupo combinado (PI+PS), Grupo Control (GC). P interacción fue calculado con 2-way ANOVA para determinar si existían diferencias significativas entre grupos Pre-intervención. Diferencias significativas entre Pre y Post intervención fueron determinadas con t-test para muestras pareadas y 1-way ANOVA se utilizó para determinar diferencias en glicemia, insulina y $\mathrm{HOMA}_{\mathbb{R}}$. Los análisis fueron ajustados por edad y asistencia al programa. Nivel de significancia fue aceptado a $\mathrm{p}<0,05$

el tiempo utilizado para recorrer $2 \mathrm{~km}$ luego de las 12 semanas de intervención $(\mathrm{p}<0,001)$, pero no los grupos PS y GC.

La presión arterial sistólica presentó una reducción significativa Post intervención sólo en el grupo PI $(\mathrm{p}=0,043)$. Los marcadores metabólicos de glicemia, insulinemia y $\mathrm{HOMA}_{\mathrm{IR}}$ fueron medidos 24 h y $72 \mathrm{~h}$ post intervención. La glicemia muestra una reducción significativa de $6,7 \%$ en el grupo PI $(p=0,011)$ y $7,4 \%$ en el grupo PS $(\mathrm{p}=0,019)$, pero no en el grupo PI+PS y GC a $24 \mathrm{~h}$ Post intervención. No se encontraron reducciones significativas en insulina y $\mathrm{HOMA}_{\mathrm{IR}}$ a $24 \mathrm{~h}$ Post intervención en ninguno de los grupos. No obstante, estas variables presentaron una reducción significativa a 72 h Post intervención en los grupos PI y PS, pero no en el grupo PI+PS y GC. La magnitud de estas reducciones fueron de 5,4\% y $6,5 \%$ en los niveles de glicemia, $18,6 \%$ y $43,4 \%$ en insulinemia, $24,1 \%$ y $55,4 \%$ en $\mathrm{HOMA}_{\mathrm{IR}}$ en el grupo PI y PS, respectivamente. El \% promedio de las reducciones (delta 24 y 72 h Post intervención) son presentados en la Figura 1.

\section{Discusión}

Los resultados de este estudio señalan que la aplicación de programas de AF de mayor intensidad, como lo son los programas PI y PS, son herramientas eficaces para la reducción de los niveles de IR en mujeres sedentarias, pre-diabéticas y con niveles de sobrepeso. También es importante destacar que el diseño de programas de AF de menor duración puede ser usado como estrategia para incrementar la práctica y adherencia a programas de AF en personas adultas con riesgo metabólico, lo cual es una necesidad considerando la realidad nacional en relación a los altos niveles de sedentarismo ( $\sim 93 \%)$, sobrepeso u obesidad ( $64 \%)$ y prevalencia de diabetes ( $\sim 9 \%)$ en mujeres ${ }^{6,7}$.

Las intervenciones propuestas en esta investigación no produjeron cambios en IMC, perímetro 
de cintura y \% de masa grasa en ninguno de los 3 grupos intervenidos. Nuestros resultados concuerdan con estudios previos donde programas similares de AF en mujeres obesas no produjeron cambios significativos en adiposidad ${ }^{19,20}$. Estos resultados podrían explicarse por el corto período de intervención.

Aunque no se detectaron cambios en marcadores de adiposidad en nuestro estudio, los programas de AF propuestos redujeron significativamente los niveles de glicemia post intervención. El control glicémico es un factor importante dentro del tratamiento de la DT2 y se asocia a complicaciones vasculares en pacientes diabéticos ${ }^{21}$. Este estudio reportó una reducción promedio de 6,1\% y 6,9\% en los niveles de glicemia post intervención en el grupo PI y PS, respectivamente. Estos cambios fueron menores a los reportados por Little y cols $^{19}$, quienes reportaron una reducción de $13 \%$ posterior a 6 sesiones de (PI) durante 2 semanas de intervención. Reducciones similares en glicemia $(15,6 \%)$ fueron reportadas por Cauza y cols ${ }^{22}$, después de una intervención con ejercicios de PS. Esta diferencia en la reducción de glicemia podría estar explicada por el tipo de pacientes intervenidos, ya que ambos estudios intervinieron a pacientes diabéticos y como se ha señalado anteriormente los beneficios de la AF son mayores en personas con riesgo metabólico (sobrepeso, sedentarios y diabéticos) ${ }^{23,24}$.

Las reducciones promedio en insulinemia (PI $\sim 16 \%$; PS $\sim 27,6)$ y $\mathrm{HOMA}_{\mathrm{IR}}$ (PI $\sim 17 \%$; PS 38), reflejan los beneficios vasculares asociados a la aplicación de ejercicios de PI y PS en mujeres con riesgo metabólico. Dichas reducciones tienen importantes implicancias clínicas debido a la asociación entre IR y el incremento en los niveles de mortalidad atribuidos a ECV ${ }^{25}$. La reducción en insulinemia y $\mathrm{HOMA}_{\mathrm{IR}}$ reportadas en nuestro estudio son similares a los beneficios obtenidos con programas de entrenamiento tradicionales, donde 150-300 min por semana de ejercicio aeróbico $\left(65 \%\right.$ del $\left.\mathrm{VO}_{2 \mathrm{MAX}}\right)$ reduce en $22 \%$ los niveles de insulinemia y mejora en $32 \%$ la sensibilidad a la insulina, después de 7 semanas de entrenamiento en mujeres con sobrepeso y antecedentes familiares de diabetes ${ }^{26}$. No obstante es importante destacar que la mayor reducción en $\mathrm{HOMA}_{\mathrm{IR}}$ asociada al grupo PS en comparación al grupo PI podría explicarse por diferencias en el volumen y tiempo de entrenamiento en nuestro estudio (Tabla 1).
Similarmente, la falta de adherencia al programa podría ser uno de los factores que podría explicar los cambios no significativos en el grupo PI+PS. Sin embargo, es importante destacar que los tres programas presentan una clara tendencia a reducir glicemia y resistencia a la insulina.

Los potenciales mecanismos mediante el cual programas de PI o PS mejoran el control glicémico y sensibilidad a la insulina resultan interesantes al considerar que no hubo cambios en las variables de composición corporal. Se ha reportado recientemente que programas de intervalo de 4 repeticiones de $30 \mathrm{~s}$, producen un gasto energético mínimo de $\sim 40-80$ kcal pero sin embargo, reducen el glicógeno muscular entre $30 \%$ y $45 \%$ mediante la vía metabólica de AMPK que juega un rol primordial en la translocación de GLUT4 y consumo de glucosa a nivel muscular ${ }^{27}$. Interesantemente el nivel de reducción en el glicógeno muscular inducido por 4 repeticiones de $30 \mathrm{~s}$ de ejercicios de PI, es similar a lo inducido por una sesión de $90 \mathrm{~min}$ de ejercicio aeróbico de intensidad moderada ${ }^{28}$. Adicionalmente, otro mecanismo que podría explicar la reducción en IR es la adaptación muscular a los programas de entrenamiento. Se ha reportado que pacientes diabéticos o con riesgo metabólico presentan una reducida capacidad de oxidación mitocondrial, ${ }^{29}$ y que intervenciones similares a las propuestas en nuestro estudio, incrementan la capacidad de oxidación mitocondrial tanto en sujetos sanos ${ }^{30}$ como con riesgo metabólico ${ }^{31}$.

Adicionalmente se detectaron cambios en presión arterial sistólica (PAS) en el grupo de intervalos, esto podría explicarse debido a que el grupo PI presentaba niveles más elevados de PAS que los otros grupos Pre intervención. Los mecanismos mediante los cuales los ejercicios PI reducen la PAS no han sido completamente elucidados, pero es posible que estén mediados por una reducción del control vascular del sistema nervioso simpático ${ }^{32}$ y a los mecanismos de vasodilatación asociados a un incremento en la producción de óxido nítrico por el endotelio ${ }^{33}$.

Es importante destacar algunas fortalezas del presente estudio, como lo fue parear según IMC y niveles de masa grasa los grupos intervenidos. Adicionalmente el diseño de los programas de ejercicio se realizó considerando protocolos de simple aplicación, y factibles de ser replicados en otros CESFAM a nivel nacional. Finalmente, se consideraron técnicas estandarizadas para 
la medición de parámetros antropométricos y metabólicos. No obstante, es importante mencionar potenciales limitaciones en el diseño del estudio que deberían ser consideraras en futuras investigaciones. Dentro de las limitaciones se encuentra el control de la ingesta calórica durante la intervención, ya que los cambios detectados en marcadores metabólicos pudieron ser afectados por cambios en patrones alimenticios, no obstante, se instruyó constantemente a los participantes no modificar su alimentación. Similarmente, no se controlaron objetivamente los niveles de actividad física durante la intervención, sin embargo, se enfatizó la no realización de actividad física fuera de los programas de intervención. Otra limitación fue la distribución de edad en los grupos, la edad promedio fue mayor en el grupo PS lo cual podría explicar por qué dicho programa presenta mayores reducciones, no obstante, la edad fue ajustada en los modelos estadísticos y no se detectaron diferencias en la magnitud y dirección de los resultados. Finalmente, la adherencia a los programas de intervenciones es otro factor de confusión, por ende los análisis fueron ajustados por nivel de asistencia.

En conclusión, los programas PI y PS son alternativas efectivas para la reducción de insulino resistencia en mujeres sedentarias, pre-diabéticas y con sobrepeso. El tiempo total de ejercicio requerido por semana para producir beneficios a nivel metabólico fue de 60 min en el grupo PI y 90 min en el grupo PS, que representan $40 \%$ y $60 \%$, respectivamente, de las recomendaciones tradicionales de AF (150 min x semana $)^{10}$. Considerando que la falta de tiempo ha sido una de las principales justificaciones para la no práctica de actividad física, creemos que esta estrategia de reducir el tiempo de ejercicio podría ser una alternativa viable para incrementar la adherencia a programas de intervención. Los programas de ejercicio presentados en esta investigación evidencian que implementar programas de menor duración en los Centros de Salud Familiar a nivel nacional es una herramienta de prescripción alternativa, fácil de implementar y eficaz en la reducción de riesgo cardiovascular asociado a la resistencia a la insulina en pacientes con riesgo metabólico.

Agradecimientos: A todos los participantes, por su compromiso con el programa. A Carmen Gloria Flores, Directora CESFAM Los Lagos y a
Leslie Ruiz, Coordinadora del Programa de Promoción de la Salud, del CESFAM, por su gestión administrativa en el proyecto.

\section{Referencias}

1. McLaughlin T, Allison G, Abbasi F, Lamendola C, Reaven G. Prevalence of insulin resistance and associated cardiovascular disease risk factors among normal weight, overweight, and obese individuals. Metabolism. 2004; 53 (4): 495-9.

2. Reaven GM. Insulin resistance: the link between obesity and cardiovascular disease. Med Clin North Am. 2011; 95 (5): 875-92.

3. Celis-Morales CA, Pérez-Bravo F, Ibanes L, Sanzana R, Hormazabal E, Ulloa N, et al. Insulin resistance in Chileans of European and indigenous descent: evidence for an ethnicity x environment interaction. PloS One. 2011; 6 (9): e24690.

4. Proper KI, Singh AS, van Mechelen W, Chinapaw MJM. Sedentary behaviors and health outcomes among adults: a systematic review of prospective studies. Am J Prev Med. 2011; 40 (2): 174-82.

5. Thorp AA, Owen N, Neuhaus M, Dunstan DW. Sedentary behaviors and subsequent health outcomes in adults a systematic review of longitudinal studies, 1996-2011. Am J Prev Med. 2011; 41 (2): 207-15.

6. Villalón GC GG, Vera SS. Evolución de la mortalidad en Chile según causas de muerte y edad: 1990-2007. Publicacion Especial. Chile: Instituto Nacional de Estadistica; 2010.

7. MINSAL. Encuesta Nacional de Salud 2009-2010. Chile: Ministerio de Salud; 2010.

8. Ahmed HM, Blaha MJ, Nasir K, Rivera JJ, Blumenthal RS. Effects of physical activity on cardiovascular disease. Am J Cardiol. 2012; 109 (2): 288-95.

9. Dumith SC, Hallal PC, Reis RS, Kohl HW, 3rd. Worldwide prevalence of physical inactivity and its association with human development index in 76 countries. Prev Med 2011; 53 (1-2): 24-8.

10. Garber CE, Blissmer B, Deschenes MR, Franklin BA, Lamonte MJ, Lee IM, et al. American College of Sports Medicine position stand. Quantity and quality of exercise for developing and maintaining cardiorespiratory, musculoskeletal, and neuromotor fitness in apparently healthy adults: guidance for prescribing exercise. Med Sci Sports Exerc 2011; 43 (7): 1334-59.

11. O'Donovan G, Blazevich AJ, Boreham C, Cooper AR, Crank H, Ekelund U, et al. The ABC of Physical Activity for Health: a consensus statement from the British 
Association of Sport and Exercise Sciences. J Sports Sci 2010; 28 (6): 573-91.

12. Reichert FF, Barros AJD, Domingues MR, Hallal PC. The role of perceived personal barriers to engagement in leisure-time physical activity. Am J Public Health 2007; 97 (3): 515-9.

13. Whyte LJ, Gill JMR, Cathcart AJ. Effect of 2 weeks of sprint interval training on health-related outcomes in sedentary overweight/obese men. Metabolism 2010; 59 (10): 1421-8.

14. ADA. Report of the Expert Committee on the Diagnosis and Classification of Diabetes Mellitus. Diabetes care 1997; 20 (7): 1183-97.

15. Marfell-Jones M, Olds, T., Stewart, A. and Carter, L. International standards for anthropometric assessment: ISAK 1st ed. Potchefstroom, South Africa: The International Society for the Advancement of Kinanthropometry (ISAK); 2006.

16. Matthews DR, Hosker JP, Rudenski AS, Naylor BA, Treacher DF, Turner RC. Homeostasis model assessment: insulin resistance and beta-cell function from fasting plasma glucose and insulin concentrations in man. Diabetologia 1985; 28 (7): 412-9.

17. Laukkanen RM, Kukkonen-Harjula TK, Oja P, Pasanen ME, Vuori IM. Prediction of change in maximal aerobic power by the 2-km walk test after walking training in middle-aged adults. Int J Sports Med 2000; 21 (2): 1136.

18. Saavedra C. Guía de actividad física para el adulto mayor. Santiago, Chile: Instituto Nacional del Deporte; 2006.

19. Little JP, Gillen JB, Percival ME, Safdar A, Tarnopolsky MA, Punthakee Z, et al. Low-volume high-intensity interval training reduces hyperglycemia and increases muscle mitochondrial capacity in patients with type 2 diabetes. J Appl Physiol 2011; 111 (6): 1554-60.

20. Gibala MJ, Little JP, van Essen M, Wilkin GP, Burgomaster KA, Safdar A, et al. Short-term sprint interval versus traditional endurance training: similar initial adaptations in human skeletal muscle and exercise performance. J Physiol 2006; 575 (Pt 3): 901-11.

21. Ceriello A. The possible role of postprandial hyperglycaemia in the pathogenesis of diabetic complications. Diabetologia 2003; 46 Suppl 1: M9-16.

22. Cauza E, Hanusch-Enserer U, Strasser B, Kostner K, Dunky A, Haber P. Strength and endurance training lead to different post exercise glucose profiles in diabetic participants using a continuous subcutaneous glucose monitoring system. Eur J Clin Invest 2005; 35 (12): 745 51.

23. Gill JMR, Malkova D. Physical activity, fitness and cardiovascular disease risk in adults: interactions with insulin resistance and obesity. Clin Sci 2006; 110 (4): 409-25.

24. Armstrong MJ, Boule NG, Sigal RJ. Exercise interventions and glycemic control in patients with diabetes. JAMA 2011; 306 (6): 607.

25. Hu G, Qiao Q, Tuomilehto J, Eliasson M, Feskens EJM, Pyorala K, et al. Plasma insulin and cardiovascular mortality in non-diabetic European men and women: a meta-analysis of data from eleven prospective studies. Diabetologia 2004; 47 (7): 1245-56.

26. Barwell ND, Malkova D, Moran CN, Cleland SJ, Packard CJ, Zammit VA, et al. Exercise training has greater effects on insulin sensitivity in daughters of patients with type 2 diabetes than in women with no family history of diabetes. Diabetologia 2008; 51 (10): 1912-9.

27. Towler MC, Hardie DG. AMP-activated protein kinase in metabolic control and insulin signaling. Circ Res 2007; 100 (3): 328-41.

28. Krssak M, Petersen KF, Bergeron R, Price T, Laurent D, Rothman DL, et al. Intramuscular glycogen and intramyocellular lipid utilization during prolonged exercise and recovery in man: a $13 \mathrm{C}$ and $1 \mathrm{H}$ nuclear magnetic resonance spectroscopy study. J Clin Endocrinol Metab 2000; 85 (2): 748-54.

29. Ritov VB, Menshikova EV, Azuma K, Wood R, Toledo FGS, Goodpaster BH, et al. Deficiency of electron transport chain in human skeletal muscle mitochondria in type 2 diabetes mellitus and obesity. Am J Physiol Endocrinol Metab 2010; 298 (1): E49-58.

30. Burgomaster KA, Howarth KR, Phillips SM, Rakobowchuk M, Macdonald MJ, McGee SL, et al. Similar metabolic adaptations during exercise after low volume sprint interval and traditional endurance training in humans. J Physiol. 2008; 586 (1): 151-60.

31. Hood MS, Little JP, Tarnopolsky MA, Myslik F, Gibala MJ. Low-volume interval training improves muscle oxidative capacity in sedentary adults. Med Sci Sports Exerc 2011; 43 (10): 1849-56.

32. Halliwill JR, Taylor JA, Eckberg DL. Impaired sympathetic vascular regulation in humans after acute dynamic exercise. J Physiol 1996; 495 (Pt 1): 279-88.

33. Halliwill JR. Mechanisms and clinical implications of post-exercise hypotension in humans. Exerc Sport Sci Rev 2001; 29 (2): 65-70. 